\title{
Análisis climático de la precipitación anual e interanual en la cuenca media del río Grande de San Ramón, Costa Rica
}

\author{
Marvin E. Quesada' ${ }^{1}$ marvin.quesada@ucr.ac.cr \& Peter R. Waylen²
}

\begin{abstract}
RESUMEN
Se investigan tres sectores ubicados en la Cuenca media del río Grande de San Ramón en Costa Rica que a pesar de encontrarse muy cerca unos de otros, éstos muestran variaciones espacio temporales en la precipitación anual e interanual. Dicha variación se atribuye a patrones de circulación atmosféricos que influyen anualmente, produciendo fuertes precipitaciones durante unos años, mientras que en otros, se da más bien una disminución. Pareciera que existe una mayor probabilidad que ocurran promedios bajos de precipitación anual durante los años El Niño y promedios altos durante los años La Niña. De esta forma, la fase cálida es más marcada en aquellos lugares de topografía plana, en valles, que tienen una mayor influencia marítima y de corrientes de viento, como son los casos de Palmares y San Ramón, no así el caso de Naranjo que se ubica en las faldas de la Sección de la Cordillera Volcánica Central. Por lo tanto, se manifiestan variaciones en distancias cortas, o sea no se muestra un comportamiento homogéneo aún en regiones pequeñas.
\end{abstract}

Palabras clave: Precipitación, ENOS, variación espacio temporal, Zona de Convergencia Intertropical, Costa Rica.

\section{Climatological analysis of the annual and interannual precipitation in the río Grande de San Ramón basin, Costa Rica}

\begin{abstract}
This project investigates three sectors located in the central basin of the río Grande San Ramón in Costa Rica. In spite of their proximity to each other, these stations show considerable variation, both temporal and spatial in intra- and interannual precipitation. This variation is attributed to regional atmospheric circulation patterns in the equatorial Pacific, producing strong precipitation during some years, and declines in others. There is a greater probability of lower annual precipitation during El Niño years and higher probabilities of large totals during La Niña years. The warm phase, El Niño, of the El Niño-Southern Oscillation phenomenon (ENSO) is more pronounced in Naranjo, which is located on the lower slope of the Central Volcanic Mountain range. The influence of regional/hemispherical cannot be regarded homogeneous in mountainous areas where local conditions may prevail.
\end{abstract}

Keywords: Precipitation, ENSO, temporal and spatial variation, Inter-tropical Convergence Zone, Costa Rica.

Recibido el 19 de noviembre de 2012, aceptado el 1 julio de 2013.

\footnotetext{
1 Departamento de Ciencias Sociales, Sede Occidente, Universidad de Costa Rica, San Ramón, Alajuela, Costa Rica.

2 Department of Geography, Universidad de Florida, Gainesville, Florida, 33611 7315, USA.
} 


\section{INTRODUCCIÓN}

En la región intertropical la precipitación es la principal variable hidroclimatológica dado que muestra una gran variabilidad no sólo anual sino también interanual, especialmente en aquellas zonas donde existe un clima tropical. Unido a lo anterior, si se toma en consideración la diversidad topográfica existente en la región centroamericana, tal es el caso de Costa Rica, hacen que el estudio del clima sea aún más complejo. Por lo tanto, investigar la relación temporal y espacial de la precipitación en una microrregión, podría ser el punto de partida o una alternativa para hacer estudios de planificación territorial, especialmente para minimizar procesos de riesgos por desastres de índole socio natural.

En los países en vías de desarrollo se hace difícil la investigación en el campo climático, dado el poco interés o la falta de recursos económicos para instalar equipos de medición de elementos del clima como son las estaciones meteorológicas. De ahí que encontrar largos registros climatológicos es muy difícil. Esta situación es aún más grave cuando se trata de regiones rurales, en especial en aquellas donde no existe ningún interés en desarrollar proyectos de riego, hidroeléctricos, eólicos, o de monitoreo ambiental, entre otros que necesiten de datos meteorológicos. En vista de la variación climática que ha estado ocurriendo en algunas regiones del mundo y ante la necesidad de disponer de energías alternativas, dado los costosos precios de los combustibles fósiles, es imperativo conocer el clima regional.

Una posible alternativa es iniciar estudios de carácter regional utilizando los registros pluviométricos existentes, que de hecho es prácticamente lo único con que cuentan algunas regiones, particularmente de países subdesarrollados. De no ser así, la mayoría de los estudios en esta temática continuarán siendo a escala global y sólo se llevarían a cabo en países desarrollados que cuentan con registros climatológicos. En el caso de Costa Rica, algunas regiones cuentan con registros climáticos relativamente extensos que permiten realizar estudios climáticos.

La localización de Costa Rica entre el Mar Caribe y el Océano Pacífico, así como su complejidad topográfica hace que se produzcan diversas variaciones climáticas, específicamente en los promedios de precipitación anual. La cantidad de precipitación e incluso la extensión de la época lluviosa, está relacionada con las variaciones interanuales en los Océanos Pacífico y Atlántico Tropical (ALFARO \& CID 1999; WAYLEN \& QUESADA 2002; POVEDA et al. 2006). En Costa Rica existen tres vertientes claramente definidas, que son la vertiente Caribe, la Subvertiente Norte y la vertiente Pacífica. En la última, un hecho a considerar de estas interacciones se refleja no sólo en la duración e intensidad de la estación lluviosa, sino que también en la seca (FERNÁNDEZ \& RAMÍREZ 1991; WAYLEN et al. 1996a; ALFARO \& CID 1999; POVEDA et al. 2006), aspecto que se convierte en un impacto significativo en los diversos sectores socioeconómicos, como son la agricultura, la pesca, la energía hidroeléctrica, el abastecimiento de agua potable, entre otros.

La ocurrencia de las estaciones seca y lluviosa sobre la Depresión Tectónica Central de Costa Rica y específicamente sobre el área en estudio está asociada con la migración periódica de la Zona de Convergencia Intertropical (ZCIT) y a la persistencia del viento Alisio del Noreste. La primera migra periódicamente dentro de gran parte de la Zona Intertropical, y el segundo tiene su área de influencia desde el sur de México hasta Panamá (HASTENRATH 1976; GRAY 1993; ALFARO \& CID 1999; POVEDA et al. 2006). Las fluctuaciones de dichos patrones de circulación provocan variabilidades estacionales e interanuales que a su vez son entendibles dada la existencia de las teleconexiones que se producen en 
ambos océanos y que tienen repercusiones a distancias lejanas (GLANTZ 1996; WAYLEN et al. 1996a), alterando el patrón de precipitaciones de un año a otro o estacionalmente.

De acuerdo con algunas investigaciones (WAYLEN et al. 1996b; ALFARO \& CID 1999; WAYLEN \& QUESADA 2002) existe una fuerte relación entre la cantidad y distribución de la precipitación en Costa Rica y los cambios en las temperaturas superficiales del mar, las cuales asimismo se encuentran relacionadas con el evento ENOS (El Niño-Oscilación del Sur). A pesar de la existencia de los mencionados estudios sobre el ENOS en Costa Rica, son muy escasas las investigaciones a escala regional, como es el caso del área que se analiza en esta investigación.

\section{Ubicación y patrón general de precipitación}

La cuenca del río Grande de San Ramón se ubica en la Depresión Tectónica Central de Costa Rica (Fig. 1). Dada su localización, se caracteriza por presentar un régimen típico de la vertiente pacífica. No obstante, se presentan ligeras diferencias entre cada una de ellas, especialmente en cuanto a la cantidad de lluvia que precipita mensualmente. Para la realización de este análisis climático se consideraron tres estaciones meteorológicas que muestran una distribución bimodal de la precipitación, o sea con dos máximas durante la época lluviosa. La primera en mayo-junio y la segunda en septiembreoctubre, con algunas excepciones. Durante julio y agosto se presenta una recesión de las lluvias (COEN 1983; MAGAÑA et al. 1999; WAYLEN \& QUESADA 2002; POVEDA et al. 2006). Esta situación climática es también sustentada por HASTENRATH (1976, 2002), que indica que altas precipitaciones se registran durante mayo y junio sobre América Central, sucediendo un abrupto cambio a finales de junio, así como en julio y agosto, que se caracteriza por ser meses más secos y de menor nubosidad, lo cual se le ha atribuido a la intensificación de los vientos del Este y Noreste (GRANDOSO 1982; RAMÍREZ 1982; POVEDA et al. 2006).

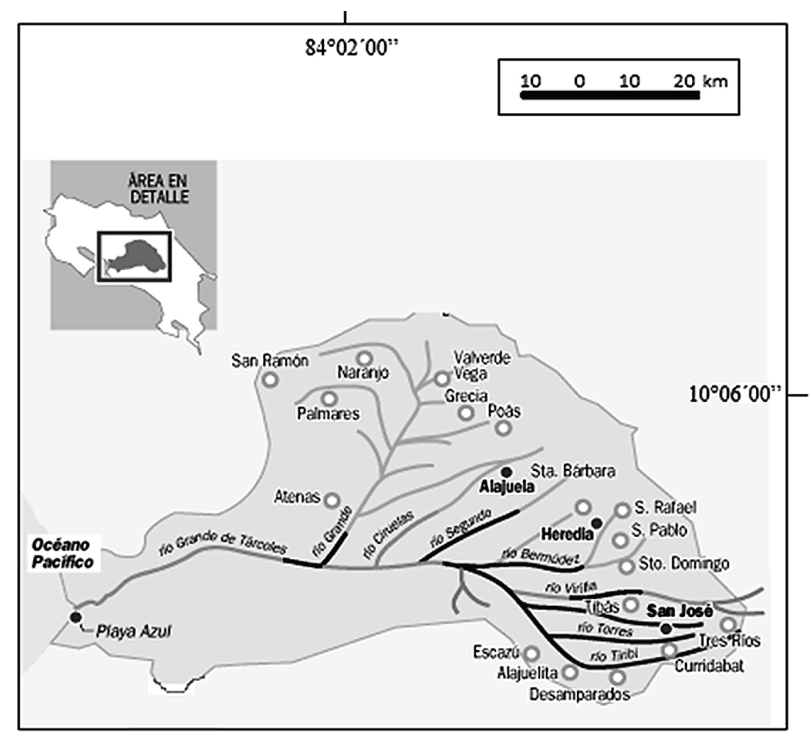

Fig. 1. Localización del área en estudio. Fuente: Laboratorio Nacional del Instituto Costarricense de Acueductos y Alcantarillados, 2000.

Fig. 1. Location study area. Source: Laboratorio Nacional del Instituto Costarricense de Acueductos y Alcantarillados, 2000. 
Como se puede observar en la figura anterior, el área en estudio lo constituye el sector Oeste de la parte media de la cuenca del río Grande de San Ramón, que a su vez se ubica en la parte Oeste de la Depresión
Tectónica Central de Costa Rica y está representada por tres centros de población o cabeceras cantonales que son Naranjo, Palmares y San Ramón (Tabla 1).

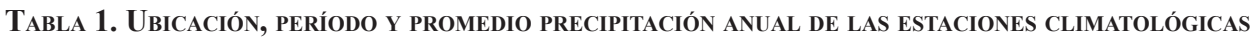
del Área EN ESTUdio. Fuente: Elaboración PROPIa CON BASE EN EL CATASTRo de LAS SERIES de PRECIPITACIÓN MEdia de Costa Rica. 2000.

TABLE 1. Location, PERIOD AND AVERAGE OF ANNUAL PRECIPITATION FOR THE Climatological STATIONS IN THE STUDY AREA. SOURCE: OWN DEVELOPMENT BASED ON THE RECORDS of PRECIPITATION OF Costa Rica, 2000.

\begin{tabular}{|c|c|c|c|c|c|}
\hline Estación & Latitud Norte & $\begin{array}{c}\text { Longitud } \\
\text { Oeste }\end{array}$ & $\begin{array}{c}\text { Elevación } \\
(\mathrm{m})\end{array}$ & $\begin{array}{c}\text { Años de } \\
\text { registro }\end{array}$ & $\begin{array}{c}\text { Promedio } \\
\text { Precipitación Anual }\end{array}$ \\
\hline Naranjo & $10^{\circ} 06^{\prime} 00^{\prime \prime}$ & $8^{\circ} 02^{\prime} 00^{\prime \prime}$ & 1042 & $\begin{array}{c}1940-1954 / \\
1960-2000\end{array}$ & 2271,6 \\
\hline Palmares & $10^{\circ} 03^{\prime} 02^{\prime \prime}$ & $8^{\circ} 02^{\prime} 00^{\prime \prime}$ & 1017 & $\begin{array}{c}1945-1948 / \\
1950-1986^{1}\end{array}$ & 1875,7 \\
\hline $\begin{array}{c}\text { San } \\
\text { Ramón }\end{array}$ & $10^{\circ} 05^{\prime} 06^{\prime \prime}$ & $8^{\circ} 28^{\prime} 38^{\prime \prime}$ & 1061 & $\begin{array}{c}1940-1973 / \\
1978-1999^{1}\end{array}$ & 1897,7 \\
\hline
\end{tabular}

${ }^{1}$ El último año de cada lugar corresponde al año en que se clausuró la estación meteorológica.

El ciclo hidroclimático anual está dominado por la migración periódica de la ZCIT, la cual controla los flujos de vientos como es el caso del Alisio sobre los océanos y sobre el sector continental, así como algunos patrones de circulación atmosféricos de carácter local y regional (POVEDA et al. 2006), los cuales vienen a definir el patrón de precipitación anual e interanual en regiones como la investigada en este caso. El resultado final de la presencia de la ZCIT es el incremento de las precipitaciones (MAGAÑA et al. 1999; HASTENRATH 2002; MURPHY et al. 2013). Aunque también se debe a la incursión de vientos provenientes del Oeste (RAMÍREZ 1982). Unido a estos patrones atmosféricos que influyen sobre el área en estudio, también existen condiciones orográficas que son las causantes de diferencias en los niveles de precipitación entre un lugar y otro.
Por otro lado, se registra un período seco definido que abarca desde diciembre hasta marzo, lo cual se debe a la disminución o ausencia en la incursión de los vientos del Oeste y a la intensificación de los vientos del Noreste. Aunque durante algunos años se pueden presentar condiciones no tan secas y se debe a la mayor persistencia de los vientos conocidos como Los Nortes, los cuales provocan condiciones inestables sobre algunos sectores de la vertiente Pacífica de Costa Rica, los cuales se visualizan por lloviznas, vientos y acompañados de una disminución en las temperaturas ambientales. Como consecuencia de la migración periódica de la ZCIT hacia el sur, se da la presencia de un período seco a mediados de año sobre la vertiente Pacífica de América Central, situación única a nivel mundial (MAGAÑA et al. 1999; POVEDA et al. 2006). Lo anterior produce una reducción de 
la precipitación en el sector en estudio, el cual durante unos años se intensifica mientras que en otros se ve reducido considerablemente.

\section{Distribución de la precipitación y su relación con ENOS}

Durante un evento ENOS (El Niño) se producen fluctuaciones en la atmósfera y en los océanos (WAYLEN \& QUESADA 2002). Algunos aspectos que se ven influenciados son la temperatura superficial del mar (TSM), la temperatura subsuperficial del mar (TsSM), la presión atmosférica, la intensidad y dirección de los vientos, la temperatura ambiental y la profundidad de la termoclina.

Estas anomalías provocan que durante unos años se produzcan condiciones meteorológicas extremas en ciertas regiones del planeta, como sequías, inundaciones, nevadas, escarcha, etc., eventos que no tienen una periodicidad definida y que al provocar alteraciones drásticas en el clima, afectan directamente la convivencia de los seres vivos e incluso repercute en la mayoría de los casos negativamente sobre el medio (GLANTZ 1996). Tales alteraciones han sido atribuidas a los cambios en las temperaturas superficiales, a lo que se le llama El Niño y La Niña y a cambios en la presión atmosférica en el nivel del mar, ambas en el Océano Pacífico, a lo que se le ha dado el nombre de Oscilación del Sur (O S). A estas oscilaciones en conjunto se le llama ENOS que ha sido uno de los principales mecanismos de variabilidad climática sobre el área tropical de América (WAYLEN \& QUESADA 2002; POVEDA et al. 2006; MURPHY et al. 2013). El Niño se refiere a un inusual calentamiento de las temperaturas superficiales del mar (TSM) sobre el océano Pacífico Este y Central. Con la consecuente disminución en los vientos Alisios. Durante un evento de El Niño se produce un cambio en la convección desde el Oeste hasta el centro del Océano Pacífico. En el caso de Costa Rica pareciera que su presencia se muestra con los cambios bruscos en la precipitación, en ciertos años dan períodos muy secos, otros muy lluviosos, entre otros. En la vertiente Pacífica cuando está presente el evento de El Niño, las precipitaciones tienden a disminuir, mientras que en la vertiente Caribe ocurre todo lo contrario, más bien existe la tendencia a aumentar los niveles de precipitación.

Al evento El Niño se le denomina la fase cálida, al ocurrir un calentamiento en las aguas oceánicas superficiales del Océano Pacífico Ecuatorial. En tanto, al evento La Niña se le ha llamado la fase fría, dado el enfriamiento de las aguas oceánicas. Por lo general, los episodios fríos producen condiciones meteorológicas contrarias a las que produce la fase cálida.

Durante la fase cálida (El Niño) las temperaturas superficiales del mar se incrementan. En tanto, durante la fase fría (La Niña), dichas temperaturas superficiales más bien disminuyen en algunos grados Celsius. Dicha variación en grados Celsius depende mucho de si se trata de un evento ENOS débil, moderado, fuerte o muy fuerte.

\section{Índice de Oscilación del Sur (IOS)}

Son varios los índices que se han propuesto para medir el patrón anual de la circulación oceánica atmosférica en el Océano Pacífico Ecuatorial que estiman las diferencias en la presión atmosférica entre Tahití y Darwin. Para esta investigación se escogió el índice de SCHNEIDER \& SCHÖNWIESE (2000), con su posterior actualización, por ser un índice muy extenso (1870-2000) y por poseer registros mensuales de la presión atmosférica a nivel del mar. Cuando este índice muestra valores negativos implica que hay pequeñas diferencias en la presión atmosférica y altas temperaturas a nivel del mar, lo que significa que se está dando una condición de El Niño. En tanto, cuando los valores son positivos y las temperaturas a nivel del mar son bajas, existe una condición de La Niña (Fig. 2). 


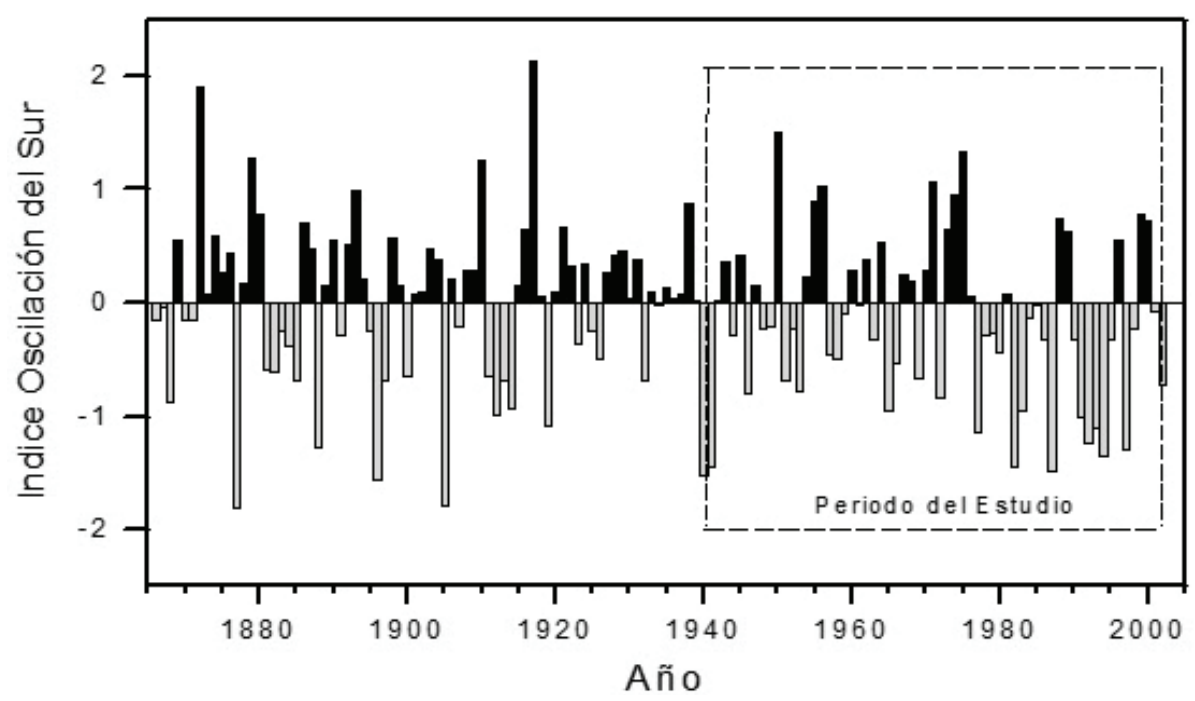

Fig. 2. Serie cronológica del Índice de Oscilación del Sur y su detalle durante el período de estudio. Fuente: Elaboración propia con base en la compilación de Schneider \& Schönwiese, 2000.

Fig. 2. Chronological series of the Southern Oscillation Index and the study period. Source: Own development based on the Schneider \& Schönwiese compilation, 2000.

\section{MATERIALES Y MÉTODOS}

Para la realización del análisis climático del área en estudio se utilizó la información disponible en la zona y que fue suministrada por el Instituto Meteorológico de Costa Rica. Como se puede observar en la tabla anterior se cuenta con tres estaciones climatológicas representativas, pero con la desventaja de que dos de las estaciones fueron cerradas. Se usó el Índice de Oscilación del Sur y las temperaturas superficiales del mar, los cuales se correlacionaron con los registros de precipitación de las tres estaciones climatológicas en análisis. Esto se hizo por períodos interanuales como anuales. Por último, se calcularon las probabilidades de ocurrencia de años secos, normales y lluviosos y su posible relación con el evento ENOS.

Con base en lo anterior se pudo hacer la identificación de años El Niño, La Niña y Normales. Para ello se escogieron los registros totales de cada una de las estaciones en estudio y se obtuvo una desviación estandarizada. Los distintos análisis realizados muestran que ha habido períodos con anomalías positivas y negativas de la presión atmosférica a nivel del mar. Es así como se efectuó un estudio que va desde 1940 hasta el 2000. Este análisis se hizo tanto desde el punto de vista anual como estacional. 
La distribución Gaussiana es adecuada para mostrar tanto períodos secos como lluviosos y es adecuada para representar períodos de precipitación anual. Esta se ajustó considerando las tres fases de la siguiente forma:

$$
F(X \geq X)=p_{1}\left[N\left(\mu_{1}, \sigma_{1}^{2}\right)\right]+p_{2} \cdot\left[N\left(\mu_{2}, \sigma_{2}^{2}\right)\right]+p_{3},\left[N\left(\mu_{3}, \sigma_{3}^{2}\right)\right]
$$

Esta expresión explica como se hizo la comparación de las estaciones meteorológicas y como se obtienen los promedios y las desviación estándar de cada una de las muestras en estudio. $\mathrm{P}_{1}$, $\mathrm{P}_{2 \mathrm{y}} \mathrm{P} 3$ que constituyen las tres estaciones climatológicas en estudio.

La precipitación anual de cada una de las fases se suma y de ahí se obtiene el promedio, la variancia y se analiza el comportamiento de la fase cálida (El Niño), la fase fría (La Niña) y la fase neutral (años normales). Se utilizó la clasificación del ENOS del Center for Ocean-atmospheric Prediction Studies (COAPS) de año 2011.

$$
t_{\text {calc }}=\frac{\left(\bar{x}_{1}-\bar{x}_{2}\right)-D_{0}}{\sqrt{S_{P}^{2}\left(\frac{1}{n_{1}}+\frac{1}{n_{2}}\right)}}
$$

$$
s_{p}^{2}=\frac{\left(n_{1}-1\right) s_{1}^{2}+\left(n_{2}-1\right) s_{2}^{2}}{\left(n_{1}-1\right)+\left(n_{2}-1\right)}
$$

$$
=\frac{\left(n_{1}-1\right) s_{1}^{2}+\left(n_{2}-1\right) s_{2}^{2}}{n_{1}+n_{2}-2}
$$

$$
t_{\text {crit }}=\begin{aligned}
& \left(w_{1} t_{1}+w_{2} t_{2}\right) /\left(w_{1}+w_{2}\right) \text { donde } w_{i}=s_{i}^{2} / n_{i} \\
& t_{i}=t_{1-\alpha / 2, n_{i}-1}
\end{aligned}
$$

Se aplicó una prueba de distribución modificada Kolmogorov-Smirnov con un nivel de confianza del 0,05 . Tanto el promedio como la variancia se calcularon para cada una de las tres condiciones oceánicas atmosféricas, de modo que de cada registro se obtuviera una prueba $t$ con dos colas, con la idea de aprobar o rechazar una hipótesis nula. La prueba $t$ se usó para verificar las diferencias en los promedios y las variancias con el fin de determinar sí los registros de lluvias tienen una distribución normal. 
Una forma de tratar de visualizar posibles diferencias regionales en la precipitación anual entre las tres ciudades en estudio durante las fases del ENOS, consiste en comparar las poblaciones de las variancias entre las tres estaciones. Ésta se hace por medio del método (Prueba $F$ Fisher) que es usada para probar una hipótesis alternativa de que la población de las variancias difiere entre ellas, o para comprobar una hipótesis nula de que ambas poblaciones de variancias son iguales. Las poblaciones de variancias son desiguales cuando los resultados muestran una $F$ grande o pequeña.

En segundo plano, se establecieron las diferencias estacionales. Para ello se subdividieron los meses del año, tomando como referencia meses secos, meses en transición, de recesión de lluvias y meses lluviosos.

\section{RESULTADOS Y DISCUSIÓN}

Con base en los índices de las temperaturas superficiales y de las presiones atmosféricas en los océanos, se puede hacer la identificación de años El Niño y La Niña. Para llevar a cabo el estudio se escogió los registros totales de cada una de las estaciones en estudio y se relacionaron con el IOS. Las figuras demuestran que ha habido períodos en que varía la presión atmosférica a nivel del mar (IOS) desde 1940 hasta el 2000.

Como se puede observar en la figura 3, la relación entre las variaciones en el IOS y los registros de precipitación en Naranjo no muestran una periodicidad en la ocurrencia de fases frías y cálidas. Es así como al considerar los promedios anuales de precipitación se encontró que los años de 1942, 1954, 1964, 1967, 1970, 1971, 1973 , 1975, 1988, 1998 tenían anomalías que se relacionan con los eventos fríos. En tanto, años como 1965, 1969, 1982, 1986, 1991 y 1997 presentaron anomalías durante los episodios cálidos.
La estación de Palmares difiere de la de Naranjo en cuanto a las anomalías anuales en la ocurrencia de episodios fríos y cálidos. Aunque ha ocurrido una menor cantidad de episodios fríos, cuando éstos están presentes han provocado grandes estragos ambientales, tal es el caso de fuertes inundaciones. Por ejemplo, en los años 1944, 1964 y 1979 se dieron altos promedios anuales de precipitación en la zona en estudio, sobrepasando los promedios de otros lugares caracterizados como lugares muy lluviosos en Costa Rica.

$\mathrm{Al}$ analizarse el comportamiento climático de cada uno de los períodos estacionales a nivel interanual se tiene que los períodos secos y de transición no mostraron anomalías relevantes. Durante el período de Mayo-Junio (MJ) dentro de los eventos fríos se destaca el año 1944 con varias anomalías. De la figura 4 se vislumbra un fuerte indicio de que hay una mayor presencia de anomalías especialmente de la fase cálida durante los períodos de JulioAgosto y Septiembre-Octubre (JA y SO).

En el asentamiento de San Ramón se presentó un comportamiento muy similar para todos los períodos en estudio, con ligeras excepciones. También se visualiza muy poca influencia de ambas fases durante el período seco y el de transición, siendo más bien el período de JA, el que muestra una mayor presencia de anomalías, durante ambas fases (Fig. 5).

Por lo tanto, pareciera que existe una asociación entre el evento El Niño y la merma en las precipitaciones, así como un aumento de las lluvias durante La Niña en las ciudades de Naranjo, Palmares y San Ramón. Unido a ello se tiene que a pesar de la cercanía de los lugares estudiados el comportamiento climático no se manifiesta en forma uniforme entre los tres lugares. Es así como para un lugar existen unos años que son secos y para el vecino no ocurre exactamente lo mismo. De igual forma, 
entre los períodos estacionales tampoco se manifiesta una homogeneidad en el comportamiento de las lluvias.

\section{Características de probabilidad en la precipitación anual}

La distribución de probabilidad normal provee un ajuste razonable tanto para la precipitación interanual como para los totales anuales en los tres períodos en los que se presentan lluvias para cada uno de los sectores en estudio. Para esta investigación se ha establecido que un $33 \%$ de los datos observados de precipitación constituyen años con niveles altos de precipitación y el $66 \%$ de los datos observados lo conforman años de escasa precipitación. En los tres sectores en estudio ha habido eventos extremos tanto durante La Niña (Fase Fría) como durante El Niño (Fase Cálida).
Si se analiza de acuerdo a cada uno de los períodos designados en esta investigación se puede deducir lo siguiente: durante los meses secos, como es obvio, es más propenso encontrar la influencia de eventos cálidos (WAYLEN \& QUESADA 2002). Una situación muy similar ocurre durante el período de noviembre-diciembre (ND) Durante la primera máxima de lluvia, mayo-junio (MJ) no se nota la presencia ni de eventos fríos ni cálidos, más bien hay una tendencia a eventos normales. En tanto que durante los meses de julio-agosto y septiembre-octubre se visualiza una mayor variabilidad entre períodos lluviosos y secos, siendo éstos precisamente los meses donde pareciera que existe mayor efecto el ENOS en cuanto a la cantidad de precipitación que cae en los tres sectores en análisis.

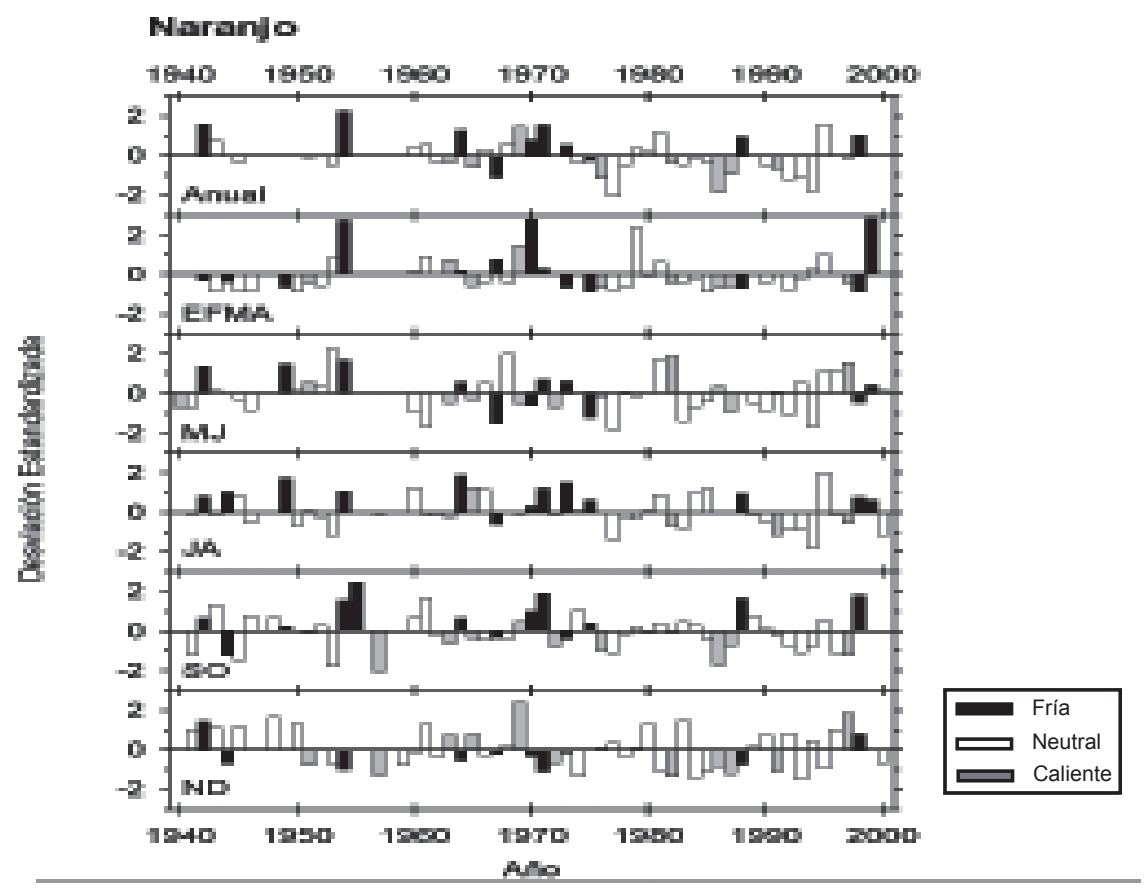

Fig. 3. Fases Fría, Neutral y Caliente, tanto anuales como interanuales en Naranjo. Fuente: Elaboración propia con base en IOS y los registros de precipitación de la Estación Beneficio Pilas, Naranjo, suministrada por el IMN de Costa Rica.

Fig. 3. Cold, Neutral and Warm Phases, annual and interannual in Naranjo. Source: Own development based on SOI and records of precipitation of Beneficio Pilas Station Naranjo, giving by the IMN of Costa Rica. 


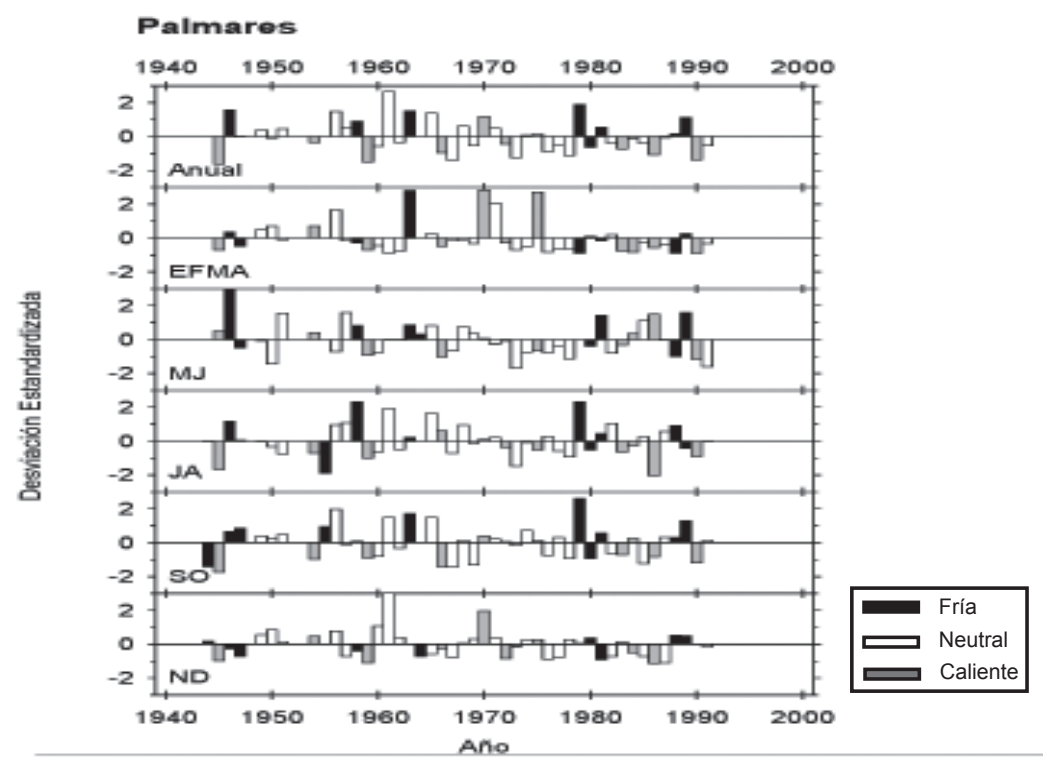

Fig. 4. Fases Fría, Neutral y Caliente, tanto anuales como interanuales en Palmares. Fuente: Elaboración propia con base en IOS y los registros de precipitación de la Estación Palmares, suministrada por el IMN de Costa Rica.

Fig. 4. Cold, Neutral and Warm Phases, annual and interannual in Palmares. Source: Own development based on SOI and records of precipitation of Station Palmares, giving by the IMN of Costa Rica.

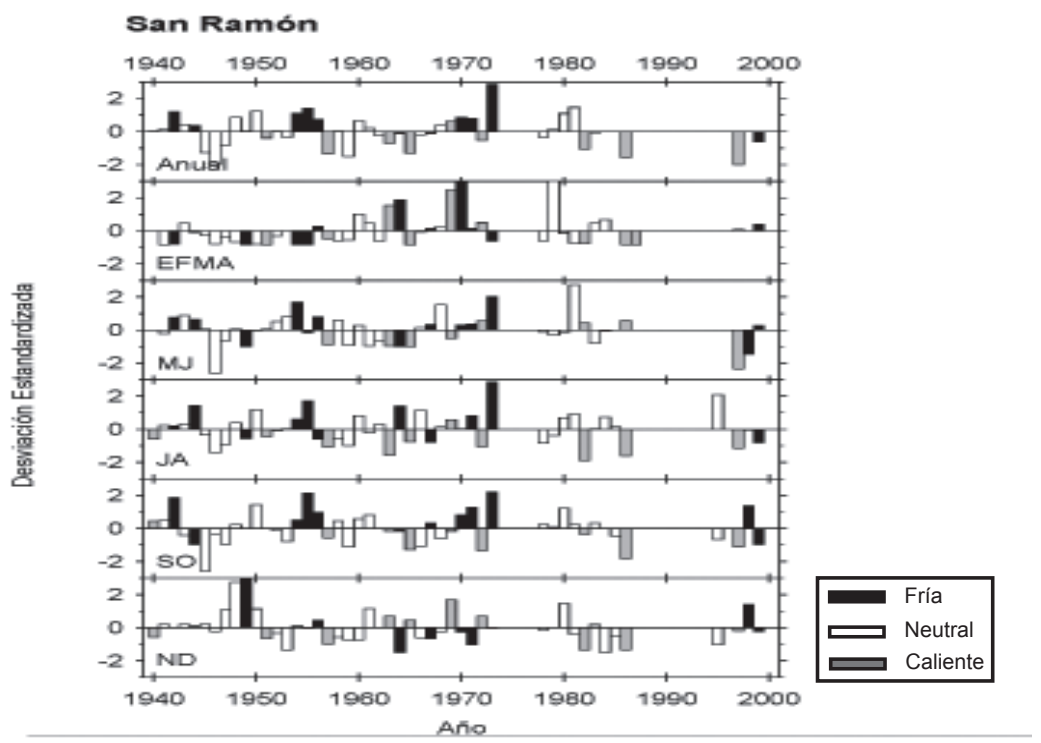

Fig. 5. Fases Fría, Neutral y Caliente, tanto anuales como interanuales en San Ramón. Fuente: Elaboración propia con base en IOS y los registros de precipitación de la Estación San Ramón, suministrada por el IMN de Costa Rica.

Fig. 5. Cold, Neutral and Warm Phases, annual and interannual in San Ramón. Source: Own development based on SOI and records of precipitation of San Ramón giving by the IMN of Costa Rica. 
Probabilidad de excedencia bajo las diferentes condiciones oceánicas atmosféricas

En las figuras 6, 7 y 8 se muestran las distribuciones de probabilidad de la precipitación anual para los tres sectores en estudio (estaciones meteorológicas se ubican en las tres principales ciudades de la cuenca media del río Grande de San Ramón). Se observa también la preponderancia de años secos asociados con la fase cálida y lluvias excesivas durante la fase fría del ENOS para todas las ciudades, siendo Naranjo el lugar con los mayores porcentajes de precipitación anual.
Tutal de fases

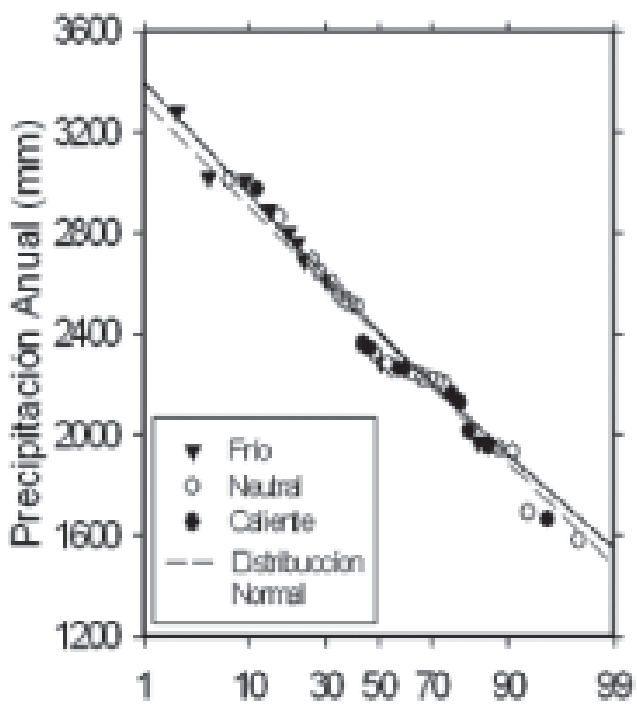

BNos

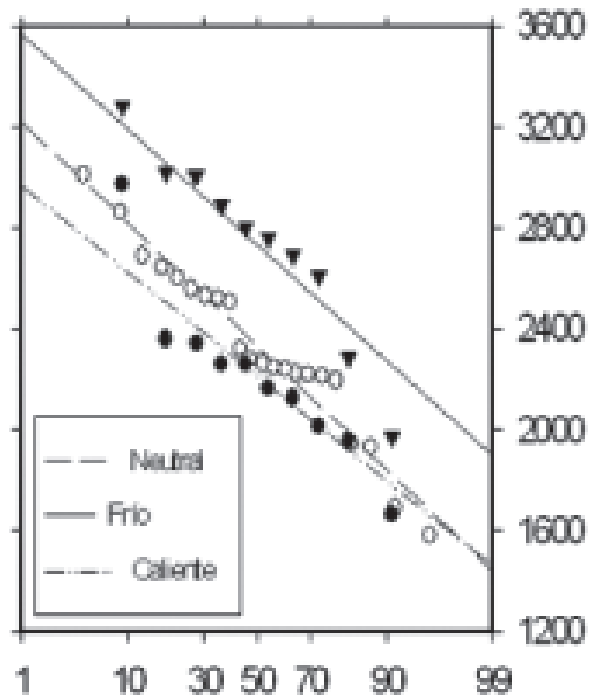

1200

\section{Prchablichd de Exoedencia}

Fig. 6. Probabilidad de excedencia de la precipitación anual en Naranjo.

Fig. 6. Probability of exceedance of the annual precipitation in Naranjo 
Total detaes

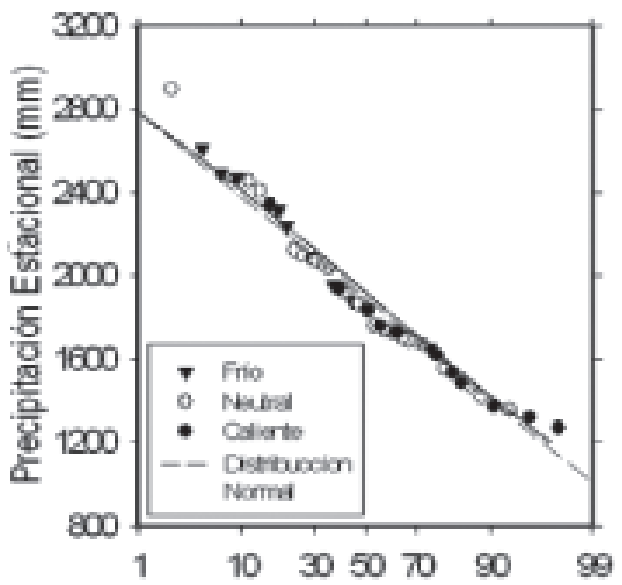

BNos

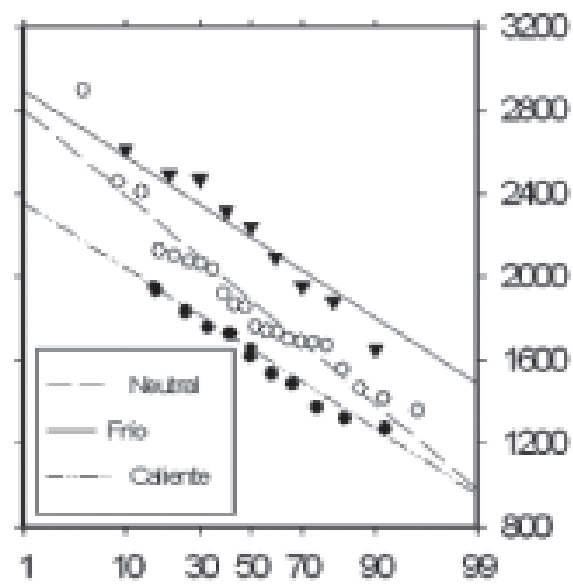

2000

2400

2000

1600

1200

\section{Prchabilichad de Excodancia}

Fig. 7. Probabilidad de excedencia de la precipitación anual en Palmares.

Fig. 7. Probability of exceedance of the annual precipitation in Palmares.

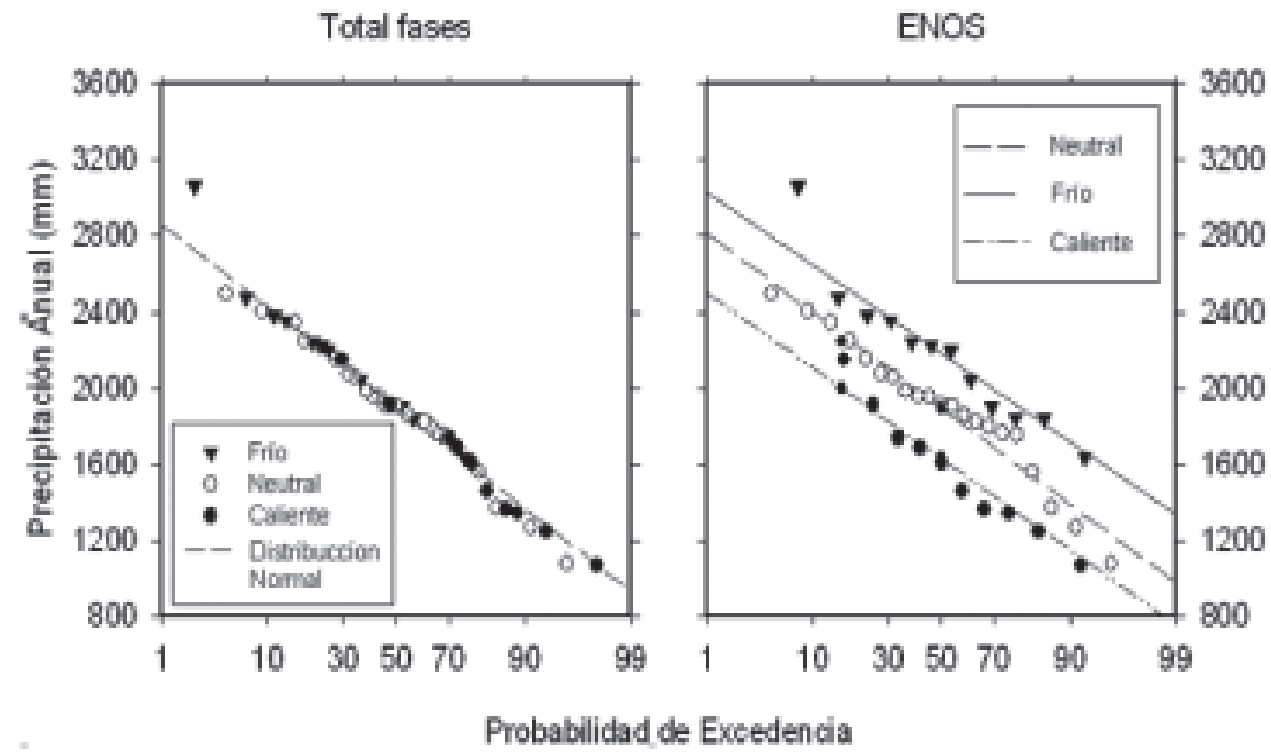

Fig. 8. Probabilidad de excedencia de la precipitación anual en San Ramón. Fuente: Elaboración propia con base en los registros de precipitación proporcionadas por el IMN.

Fig. 8. Probability of exceedance of the annual precipitation in San Ramón. Source: Own development based on precipitation records provided by the IMN. 
Aunque las tres figuras muestran comportamientos muy similares en Naranjo y Palmares, los años normales se asemejan más a los años de El Niño al aumentar el nivel de probabilidad, condición distinta a la de San Ramón. Además se tiene también que para Palmares, a pesar de que los años de La Niña no son tan lluviosos como en el caso de San Ramón y Naranjo, sí se muestra una fase cálida definida, situación que no se presenta en Naranjo.

La distribución de la precipitación interanual muestra que durante el período seco, los meses de Enero, Febrero Marzo y Abril (EFMA) se acumula muy poca o casi nula la precipitación sobre los tres lugares selecionados para el análisis. Durante la primera máxima de precipitación en los meses de Mayo y Junio (MJ) se inician las lluvias intensas en todos los sectores en estudio, aunque éstos no se manifiestan en forma uniforme. Por ejemplo, son superiores los niveles de precipitación en Naranjo especialmente durante La Niña. Durante este período del año se pone de manifiesto la influencia de la ZCIT mediante fuertes precipitación (POVEDA et al. 2005; DURAN 2010), así como la presencia de los vientos Oestes Ecuatoriales que ingresan por el Suroeste de la cuenca.

A mediados de año en los meses de Julio y Agosto (JA) se produce una ligera recesión de lluvias como producto de la migración de la ZCIT y la consiguiente incursión de los vientos alisios del $\mathrm{NE}$, que en lugar de producir lluvias, mas bien, inducen a un efecto de subsidencia sobre el área en estudio. Dicha situación es notoria durante El Niño con hasta un mes de días sin lluvia o a veces con ligeras llovisnas, lo cual depende también de la intensidad del evento ENOS. La sequía es más notoria en eventos fuertes del ENOS. Cuando se tiene una fase neutral y sobre todo la fase fría, la recesión de lluvias es casi nula (Fig. 9).

Durante los meses Septembre y Octubre (SO) de nuevo se nota la fuerte influencia de la ZCIT, la cual esta ubicada practicamente sobre la latitud de Costa Rica y es la encargada de producir las máximas precipitaciones del año. Estas son todavía más intensas durante la fase fría para los tres sectores en estudio. No obstante, durante la fase caliente a pesar de que disminuyen con respecto a la fase fría, estas continuan siendo la máxima precipitación durante todo el año. Por último, para el período de Noviembre y Diciembre (ND) no ocurre ninguna diferencia en los niveles de precipitación entre una fase y otra y tampoco entre los tres sectores analizados. Para ese momento la ZCIT ya se ha alejado de las latitudes de Costa Rica y más bien empiezan a incursionar los vientos alisios del $\mathrm{NE}$, que como ya se señaló, más bien registra masas de aire seco. 


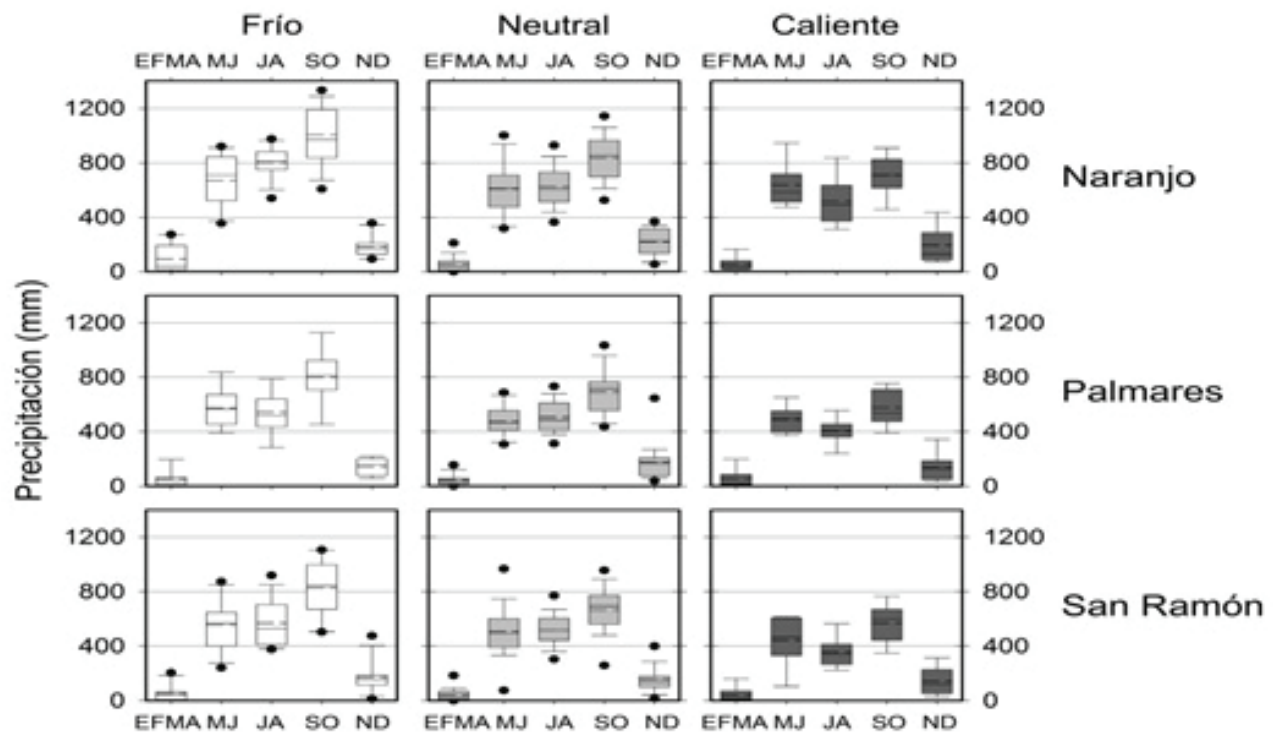

Fig. 9. Precipitación interanual durante las fases fría, neutral y caliente en en la sección media de la cuenca del río Grande de San Ramón. Fuente: Elaboración propia con base en los registros de precipitación suministrados por el IMN de Costa Rica.

Fig. 9. Interannual precipitation during the Cold, Neutral and Warm phases in the medium sector of the río Grande San Ramon basin. Source: Own development based on the precipitation records giving by the IMN of Costa Rica.

\section{CONCLUSIÓN}

Como reflexión final, se obtiene que las pruebas estadísticas aplicadas proveen un buen análisis de los registros de precipitación anual existentes en las tres ciudades que se han estudiado para visualizar la variabilidad climática de la precipitación existente en los tres sectores en estudio. A pesar de su proximidad, se aprecian diferencias regionales marcadas de un año a otro e incluso en cuanto a la cantidad de lluvia que cae sobre cada una de ellas. Se demuestra así que las diferencias en la precipitación en el sector medio de la cuenca del río Grande de San Ramón sirven para demostrar la existencia de variabilidades anuales e interanuales que están ocurriendo en dicha región.

De acuerdo con los resultados de la investigación, existe una mayor probabilidad de que ocurran promedios bajos de precipitación anual durante los años El Niño y promedios altos durante los años La Niña. Pareciera que la fase cálida es más marcada en aquellos lugares de topografía plana, en valles, que tienen una mayor influencia marítima y de corrientes de viento, como son los casos de Palmares y San Ramón.

La interacción entre patrones de circulación general como son los alisios, la ZCIT, Los Nortes y otros de carácter local como son la incursión de brizas o masas de aire locales junto con factores topográficos, tal es el caso de depresiones intermontanas, valles rodeados de barreras topográficas que juegan un papel importante en el control de la variabilidad, tanto anual como interanual de la precipitación. En aquellos lugares de topografía irregular y con rasgos de una mayor continentalidad, la fase cálida del ENOS es menos consistente, 
un ejemplo típico lo constituye la ciudad de Naranjo, dada su ubicación en un área muy irregular topográficamente y aunado a ello es una de las pocas ciudades de Costa Rica con rasgos de un clima continental. Estos factores facilitan que la disminución de los promedios de precipitación anual sean menos pronunciados durante los años de El Niño. En tanto que San Ramón, por su ubicación expuesta a dos depresiones por donde incursionan los vientos, se encuentra bajo condiciones muy inestables climáticamente, como se señaló anteriormente, teniendo una respuesta positiva, tanto ante el evento de La Niña como ante El Niño.

En síntesis, este estudio provee suficientes evidencias cuantitativas para probar la hipótesis que el patrón de precipitación en el área estudiada está mostrando variaciones, tanto anuales como interanuales. Esto está ocurriendo a consecuencia de las diferencias en las TSM en ambos océanos, el Pacífico y el Atlántico. Además de eso, se ha demostrado que aún en pequeños sectores como el que se estudió se pueden encontrar diferencias marcadas de un lugar a otro.

\section{AGRADECIMIENTOS}

Se agradece al Instituto Meteorológico Nacional de Costa Rica por su amabilidad al suministrar parte de los registros climáticos existentes sobre el área en estudio. Similarmente, se agradece a la Vicerrectoría de Investigación de la Universidad de Costa Rica por el apoyo brindado mediante la financiación parcial del proyecto $\mathrm{N}^{\circ} 540-\mathrm{A} 3-037$.

\section{REFERENCIAS}

ALFARO, E. \& L. CID, 1999. Análisis de las Anomalías en el Inicio y Término de la Estación Lluviosa en Centroamérica y su Relación con los Océanos Pacífico y Atlántico Tropical. Meteorology Oceanographic. 6 (1): 1-13.
CAVIEDES C., 1984. El Niño 1982-1983. American Geographical Society. 74: 288-289.

COEN, E., 1983. The Climate of Costa Rica. Chapter 3. Costa Rica Natural History. University of Chicago Press. 383 pp.

COAPS, 2012. ENSO Index According to JMA SSTA (1868-present). Florida, Center for Ocean-Atmospheric Prediction Studies. The Florida State University. Disponible en: http://coaps.fsu.edu/jma.shtml.

DURAN A., J. GEMENO, J.A. AMADOR \& R. NIETO, 2010. Moisture Sources for Central America: Identification of Moisture Sources using a Lagrangian Analysis Technique. Journal of Geophysical Research. 115: 5-103.

FERNÁNDEZ, W. \& P. RAMÍREZ, 1991. El Niño la Oscilación del Sur sus Efectos sobre Costa Rica: Una revisión. Tecnología en Marcha. 11: 3-11.

GLANTZ M., 1996. Currents of Change: El Niño's Impacts on Climate and Society. 1era edición. Cambridge. Cambridge University Press. 253 pp.

GRANDOSO, H., 1982. Características de la Atmósfera Libre sobre Costa Rica y su Relación con la Precipitación. Informe semestral, I.G.N, Costa Rica. Enero-Junio, pp. 11-52. San José, Costa Rica.

GRAY G., 1993. Regional Meteorology and Hurricanes. Climate Change in the Intra-American Sea. G. Maul, Ed. Eduard Arnold, 112. 87-99.

HASTENRATH S. L. 1976. Variations in Low Latitude Circulation and Extreme Climatic Events in the Tropical Americas. Journal of the Atmospheric Sciences. 33: 202-215.

HASTENRATH S. L., 2002. The Intertropical Convergence Zone of the Eastern Pacific Revisited, International Journal of Climatology. 22: 347-356. 
HERRERA W., 1989. Clima de Costa Rica. EUNED. San José, Costa Rica. 118 pp.

MAGAÑA, V., J.A. AMADOR \& S. MEDINA, 1999. The Mid-summer Drought over Mexico and Central America. Journal of Climate. 12(6): 1577-1588.

MEJÍA, J.F. \& G. POVEDA, 2005. Atmospheric Features of Mesoscale Convective Systems over Colombia during 1998 using TRMM and the 40-year NCEPNCAR Reanalysis Data Sets (in Spanish). Rev. Academia Colombiana de Ciencias Exactas, Físicas y Naturales. 29 (113): 495-514.

MURPHY J., K. GEORGAKAKOS \& E. SHAMIR, 2013. Climatological Analysis of December Rainfall in the Panama Canal Watershed. International Journal of Climatology., doi: 10.1002/joc.3694.

POVEDA G., O.J. MESA, L.F. SALAZAR, P.A. ARIAS, H.A. MORENO, S.C. VIEIRA, P.A.GUDELO, V.G. TORO \& J.F. ALVAREZ, 2005. The Diurnal Cycle of Precipitation in the Tropical Andes of Colombia. Monthly Weather Review. 133 (1):, 228-240.

POVEDA G., P.R. WAYLEN \& R.S. PULWARTY, 2006. Annual and Inter-annual Variability of the Present Climate in Northern South America and Southern Mesoamerica. Palaeogeography, Palaeoclimatology, Palaeoecology. 234: 3-27.
RAMÍREZ, P., 1982. Estudio meteorológico de los Veranillos en Costa Rica. Nota Investigación $\mathrm{N}^{\circ}$ 5. Instituto Meteorológico Nacional, Ministerio de Agricultura y Ganadería. San José, Costa Rica. 48 pp.

SCHNEIDER, U. \& C. SCHÖNWIESE, 1989. Statistical characteristics of El NiñoSouthern Oscillation and North Atlantic Oscillation indices. Atmosfera. 22: 34-47.

WAYLEN P.R., M.E. QUESADA \& C.N. CAVIEDES, 1994. The Effect of El Niño Southern Oscillation on Precipitation in San José, Costa Rica. International Journal of Climatology. 14: 559-568.

WAYLEN, P.R., M.E. QUESADA \& C.N. CAVIEDES, 1996a. Temporal and Spatial Variability of Annual Precipitation in Costa Rica and the Southern Oscillation. International Journal of Climatology. 16:173-193.

WAYLEN, P.R., M.E. QUESADA \& C.N. CAVIEDES, 1996b. Interannual Variability of Monthly Precipitation in Costa Rica. Journal of Climate. 9: 2606-2613.

WAYLEN P. R. \& M.E. QUESADA, 2002. The Effect of Atlantic and Pacific Sea Surface Temperature on the MidSummer Drought of Costa Rica. Journal of Environmental Change and Water Sustainability. Instituto Pirenaico de Ecología, Zaragoza. España. 197-209. 